\title{
Discusión sobre sociología de la literatura
}

En el extremo, a veces parecería que puede haber tantas sociologías de la literatura como sociólogos de la literatura (e incluso más sociologías que sociólogos, si pensamos que muchos inves-

Quisiera discutir algunos puntos sobre sociología de la literatura. tigadores, sin proponérselo, efectúan aportes a este impreciso terreno de indagaciones). Por lo tanto, ante este panorama, al menos como medida clasificatoria binaria, la distinción entre las

Dada la aparente vaguedad de la sub-disciplina, me parece útil pensar una división del área de estudios a partir de los debates de los últimos veinte años sobre la literatura mundial y el futuro de las literaturas comparadas: si bien, en general, los aportes de Casanova y Moretti son leídos y valorados de manera conjunta por la sintonía de sus propuestas — la aproximación a la literatura mundial por medio de un enfoque sistémico o estructural, en que el mundo literario es entendido como uno y desigual-,${ }^{1}$ al mismo tiempo sus lineamientos teóricos derivan en dos programas distintos de sociología de la literatura: ¿ésta consiste en una sucesión de luchas que se desenvuelven en un espacio literario, regidas por una serie de estrategias que toman los agentes para posicionarse y ser reconocidos en dicho ámbito (y cuya metodología, por ende, se basa en estudiar las prácticas de los agentes para legitimarse) o, más bien, se trata de una sociología de las formas literarias y su evolución (y cuya metodología, por más vuelta renovadora que se le quiera dar, se centra en leer textos y analizarlos críticamente)?.

1 Pascale Casanova, La República mundial de las Letras, Barcelona, Anagrama, 2001; Franco Moretti, "Conjectures on World Literature", en New Left Review, $n^{\circ} 1,2000, p p .54-68$. Por dar algunos ejemplos de la recepción articulada de ambos en la Argentina, pensemos en: María Teresa Gramuglio, "El cosmopolitismo de las literaturas periféricas", en CeLeHis. Revista del Centro de Letras Hispanoamericanas, $n^{\circ} 19$, 2008 , pp. 159-172 (y, en un número anterior de la misma revista, hay otro aporte dedicado a Casanova y Even-Zohar: María Teresa Gramuglio, "Literatura argentina y literaturas europeas. Aproximaciones a una relación problemática", en CeLeHis. Revista del Centro de Letras Hispanoamericanas, $n^{\circ} 16,2004$, pp. 11-27); Alejandro Dujovne y Diego García, "Introducción a la 'Literatura mundial"', en Políticas de la Memoria, $n^{\circ}$ 10/11/12, 2009-2011, pp. 31-37; Marcelo Topuzian, "La literatura mundial como provocación de los estudios literarios", en Chuy. Revista de estudios literarios latinoamericanos, $n^{\circ} 1,2014 a, p p .94-138 ;$ Marcelo Topuzian, "Introducción: entre literatura nacional y posnacional", en Marcelo Topuzian (comp.), Tras la nación: conjeturas y controversias sobre las literaturas nacionales y mundiales, Buenos Aires, Editorial Universitaria de Buenos Aires, 2017, pp. 9-65.

2 Desde luego, hay otras formas en que se presentan estas posiciones presociologías de la literatura de Casanova y Moretti puede servirnos para establecer: se estudian las prácticas de los agentes o se estudian los textos. Vale aclarar: ¿acaso alguien dijo que se trata de enfoques excluyentes? Por supuesto que no. ${ }^{3}$

\section{|}

Más allá de los programas de Casanova y Moretti o, mejor dicho, justamente pasando por ellos, tenemos un hecho doblemente conflictivo para la sociología de la literatura, pues esta implica dos grandes áreas de problemas e imprecisiones en torno a los vocablos "sociología" y "literatura". Ambos suponen un dominio determinado de indagación, con su objeto de estudio correspondiente ("la sociedad" y "la literatura", pongamos), con su metodología y sus presupuestos teóricos. Esta doble pertenencia es, por cierto, doblemente conflictiva, si pensamos que cada disciplina de base arrastra sus propios problemas conceptuales. En la Argentina ha tendido a predominar una sociología de la literatura de orientación bourdieusiana, ${ }^{4}$ particularmente a partir de Las

suntamente dicotómicas: como críticas "internas" y "externas" o como escuelas francesa e inglesa. Cada una de estas clasificaciones representa un enfoque distinto del problema, aunque hay ciertas analogías y, no sin cierta cautela, podríamos identificar a Casanova y Moretti también a partir de estas oposiciones: la primera resultaría ligada, vía Bourdieu, a la crítica "externa" y francesa; el segundo, vía Jameson, a la crítica "interna" e inglesa.

3 ¿Hace falta aclarar que una clasificación no es verdadera ni falsa, sino útil o inefectiva? Hace un tiempo que considero que esta violenta partición es particularmente útil, no porque yo la postule, sino porque creo que todo trabajo de sociología de la literatura puede ser sometido (en el mejor y en el peor sentido) a ella. Hagan la prueba.

4 Pensemos, por ejemplo, en las referencias de algunos ensayos de Tabarovsky: cuando confronta con la sociología de la literatura, habla taxativamente de una sociología de orientación bourdieusiana, aunque no tenga necesidad de mencionar al propio Bourdieu (Damián Tabarovsky, Literatura de izquierda, Rosario, Beatriz Viterbo, 2004, pp. 10, 39, 59 y 94). Este ejemplo es apenas uno entre incontables presencias en el fluir de la vida académica y universitaria: programas de asignaturas de grado 
reglas del arte..$^{5}$ Sin embargo, si quisiéramos comenzar con el doble carácter problemático en torno a "lo social" y "lo literario", quizá un mejor y más sensato punto de partida para un programa de sociología de la literatura sea el texto "Lo literario y lo social", de Robert Escarpit, ${ }^{6}$ cuya figura ha sido opacada, precisamente, por el predominio colonizador de la teoría bourdieusiana. ${ }^{7}$

Ahora bien, tomar a Escarpit como punto de partida no significa ensalzarlo y situarlo como dogma sagrado; significa sólo lo que acabo de decir: tomarlo como punto de partida. ${ }^{8}$ Por ejemplo,

y posgrado, marcos teóricos de ponencias, artículos y tesis, etcétera. No hace falta demostrar la colonización de buena parte de las ciencias sociales y humanísticas por parte de la teoría de Bourdieu: uno sencillamente se topa con ella todo el tiempo. Pero, si quieren referencias, para una aproximación general a la recepción de Bourdieu en la Argentina, nos remitimos a dos trabajos: Ana Teresa Martínez, "Lecturas y lectores de Bourdieu en la Argentina", en Prismas: revista de historia intelec tual, n 11, 2007, pp. 11-30; Denis Baranger, "La recepción de Bourdieu en Argentina", en Desarrollo Económico, n 197, 2010, pp. 129-146. Para tomar dimensión de Bourdieu como el sociólogo más citado del mundo en los últimos años: Marco Santoro, "Putting Bourdieu in the Global Field. Introduction to the Symposium", en Sociologica, $n^{\circ} 2,2008$, pp. 1-32.

5 Pierre Bourdieu, Las reglas del arte. Génesis y estructura del campo literario, Barcelona, Anagrama, 2005.

6 Robert Escarpit, "Lo literario y lo social", en Hacia una sociología de hecho literario, Madrid, Edicusa, 1974, pp. 11-43. No está de más recordar que dicho libro se encuentra citado en el fundacional estudio de Altamirano y Sarlo (Carlos Altamirano y Beatriz Sarlo [eds.], Literatura y sociedad, Buenos Aires, Centro Editor de América Latina, 1977), aunque, a la hora de seleccionar un fragmento, eligen uno de un libro anterior y menos maduro, Sociología de la literatura (Robert Escarpit, Sociología de la literatura, Buenos Aires, Fabril Editora, 1962). Luego, en un trabajo posterior, traen a cuenta un par de ideas del francés: por un lado, la preocupación por el problema de la "supervivencia" de las obras literarias y, por otro, el abordaje de los hechos literarios a partir de las prácticas externas por parte de los lectores (Carlos Altamirano y Beatriz Sarlo, Literatura/Sociedad, Buenos Aires, Hachette, 1983, pp. 95-96 y 105-106, respectivamente). Más allá de estas referencias puntuales de Altamirano y Sarlo, encontramos escasas alusiones a Escarpit en nuestro país: las citas de Garasa en Literatura y sociología (Buenos Aires, Troquel, 1973 pp. 18,19 y 22) y algunas otras menciones al pasar, como observamos en un breve texto de María Elisa Cevasco ("Literatura, sociología de la literatura", en Carlos Altamirano [dir.], Términos críticos de sociología de la cultura, Buenos Aires, Paidós, 2002, p. 162), en un artículo de Hernán Vanoli ("Pequeñas editoriales y transformaciones en la cultura literaria argentina", en Apuntes de Investigación del CECYP, $n^{\circ} 15,2009$, p. 161) o en un libro de Daniela Szpilbarg (Cartografía argentina de la edición mundializada. Modos de hacer y pensar el libro en el siglo XXI, Temperley, Tren en Movimiento, 2019, p. 25).

$7 \quad$ No es menor el hecho de que Bourdieu, en Las reglas del arte, casi no mencione a Escarpit, salvo por una casi inadvertida nota a pie de página en que hace quedar a Escarpit como un tonto, al adjudicarle la práctica de una "sociología que vincula directamente las características de las obras con la procedencia social de los autores" (op. cit., p. 344).

8 Desde luego, hay otros pensadores y otras tradiciones de sociología de la literatura que pueden contener premisas perfectamente válidas para iniciar una reflexión. Por el evidente motivo de la finitud del sujeto empírico que escribe estas páginas, me limito a señalar un posible punto de partida (no el único, desde luego, pero sí uno que resulta significativo, pues resalta la doble problemática de "lo social" y "Lo literario"). Otras opciones de desarrollos teóricos que podrían funcionar como puntos de partida alternativos: las formulaciones de Lukács (Sociología de la literatura, Madrid, Península, 1966) o incluso las discusiones que se dan entre distintos teóricos europeos y que incluyen a Barthes, Goldmann y a los propios Lukács y Escarpit, entre otros. Roland Barthes y otros, Literatura y sociedad. Problemas de metodología en sociología de la literatura, Barcelona, Martínez Roca, 1969; Lucien Goldmann y otros, Sociología de la creación literaria, Buenos Aires, Nueva Visión, 1971; Lu- quisiera apartarme de su idea en torno a que, en la sociología de la literatura, la sociología vendría a representar el método, mientras que la literatura tendría reservado el lugar de objeto. ${ }^{9}$ Si partimos de asumir a la sociología como método y a la literatura como objeto, ya desde el comienzo ingresaríamos en un itinerario estrecho, reduccionista y aporético. No quiero decir con esto que la sociología de la literatura no pueda incluir, entre sus variantes, un abordaje en que tengamos a la sociología como método y a la literatura como objeto; lo que sí quiero rechazar es que tal sea la única modalidad de esta área de estudios.

A continuación, vuelvo al punto de partida de Escarpit y amplío lo que veo como el doble problema fundacional de la sociología de la literatura, a través de las extensas arenas (¿o debería decir los oscuros pantanos?) de "lo social" y "lo literario".

Si me remito a la tradición del pensamiento sociológico, "lo social" es un problema - y no tanto un método o una solución-. "Lo social" podría ser un contrato (y sí: los contractualistas), un hecho (Durkheim), una acción referida a otro (Weber), un modo de producción y/o una lucha (Marx y Marx; el otro, el mismo), una acción social entendida como sistema (Parsons, en su versión estructural-funcionalista), un mundo de la vida cotidiana (Schutz), un conjunto de actividades rutinarias regidas por reglas (Garfinkel), un espacio conflictivo de posiciones interrelacionadas (Bourdieu), un proceso de agenciación y estructuración (Giddens), un sistema autopoiético de comunicaciones recursivas (Luhmann), una acción comunicativa (Habermas), una red de relaciones entre actores, artefactos y discursos (Latour, por mencionar una de las últimas modas) y, si me permiten usar mi fórmula favorita y la que resuelve el fin de la lista: etcétera. Queda claro que me estoy refiriendo al núcleo de la tradición sociológica que, desde luego, está compuesta por hombres blancos, culturalmente burgueses, europeos o norteamericanos. Esto no quita que podamos (y debamos) extender las opciones de "lo social" hacia los giros decolonial, feminista, afectivo, espacial, cuantitativo, etcétera, aunque, a los fines prácticos, los abordajes emergentes sólo confirman mi punto: la inabordable ampliación de "lo social". La sociología se torna cada vez más "intransparente"10 y "lo social", entonces, se erige como un problema ante el que el investigador debe tomar decisiones. Desde luego, puede haber desarrollos sistematizadores, integradores y sintetizadores de distintas corrientes, como han intentado Parsons, Bourdieu o Habermas, entre otros, pero incluso los proyectos más "democráticos" de integración y síntesis excluyen a un remanente de autores y formulaciones sobre "lo social": hoy no es posible,

cien Goldmann, Para una sociología de la novela, Madrid, Ayuso, 1975.

9 Escarpit se refiere a una "sociología de la literatura que exige una metodología sociológica, pero una problemática literaria". Robert Escarpit, "Éxito y supervivencia literarios", en Hacia una sociología del hecho literario, Madrid, Edicusa, 1974, p. 160

10 Niklas Luhmann, Sistemas Sociales: lineamientos para una teoría general, Rubí (Barcelona)/México D. F./Bogotá, Anthropos/Universidad Iberoamericana/Pontificia Universidad Javeriana, 1998, p. 8. 
para un sociólogo, ser absolutamente democrático para con la teoría sociológica (aunque esto no implique que no debamos actuar lo más democráticamente posible en nuestros diseños teórico-metodológicos). Lo mismo vale para alguien que se auto-perciba como sociólogo de la literatura: cualquier formulación de un programa (de docencia y/o investigación) de sociología de la literatura presupone la asunción de una teoría sobre la sociedad - y explicitar la teoría de la sociedad de la que se parte debe ser incluido en tal programa, tanto como el reconocimiento de otras teorías de la sociedad que resulten marginadas-. ${ }^{11}$

Cuando pasamos a los estudios literarios, nuevamente nos topamos con una dimensión análoga respecto a lo que representa "lo social" para la sociología: el problema de la especificidad de "lo literario", pregunta que, en una iteración diabólica, atormenta y seduce a las mentes de los estudiosos de la literatura. Distintas escuelas y corrientes teóricas siguen merodeando la pregunta respecto a la especificidad de lo literario: el formalismo ruso, el marxismo, la estética de la recepción, el estructuralismo, el pos-estructuralismo, el feminismo, etcétera. "Lo literario" puede abarcar tanto un uso diferenciado del lenguaje cotidiano como uno diferenciado de los usos previos del propio discurso reconocido e instituido como literario; puede consistir en un conjunto reducido y manejable de técnicas de composición o puede implicar una serie de trabajos radicales, excesivos e "incontrolables"

11 Resulta llamativo cómo algunos sociólogos de la literatura se desligan sutilmente del problema de "lo social". Al respecto, pienso que incluso podemos hallar un punto ciego en las formulaciones fundacionales de Altamirano y Sarlo, no sólo en la compilación de artículos de otros pensadores que ya hemos mencionado, Literatura y Sociedad, sino también en sus propias elaboraciones más extensas: el útil mapa de problemas gestado en Conceptos de sociología literaria (Buenos Aires, Centro Editor de América Latina, 1980) y, especialmente, el más profundo e indispensable Literatura/Sociedad (Buenos Aires, Hachette, 1983). ¿O vamos a presuponer que todos entendemos lo mismo por "sociedad"? Este reparo no quita que ellos hayan renovado de forma vital los alcances de los estudios literarios y de la sociología de la literatura (por ejemplo, a través de la introducción de las perspectivas teóricas de Raymond Williams y del propio Bourdieu). Entonces, ¿hace falta que aclare que sus trabajos son condición de posibilidad de mi modesto y torpe planteo? A su vez, queda claro que la trayectoria posterior de ambos los aproximó a otra sub-disciplina, la historia intelectual, tal como me ha recordado el comité de la revista Políticas de la Memoria (pero, yo diría: en todo caso, la historia intelectual supone la asunción de una determinada definición de "sociedad"). Además, el comité de la revista me ha sugerido detenerme en dos preguntas que resultan altamente pertinentes, aunque no tenga respuesta (ni más espacio en este texto), por lo que al menos las dejo consignadas: "¿no habría que considerar a las familias del marxismo occidental a la hora de un relevamiento y un balance [de la sociología de la literatura]? ¿No fue la 'sociología de la literatura' el modo que encontraron Sarlo y Altamirano de hablar de marxismo en los años de la dictadura?". En cuanto a esta última pregunta, es una buena hipótesis que, supongo, puede ser respondida con una consulta a los propios Altamirano y Sarlo. En cuanto a la primera pregunta: por supuesto que sí (y considero que parte de esa inclusión está contenida en mi diagnóstico que incluye al marxismo tanto en sus formulaciones sobre "lo social" como sobre "lo literario"). Entre otras opciones de atisbo de respuesta, podríamos retomar la existencia de generaciones de sujetos marxistas con enfoques teóricos centrados en la "famosa" superestructura, tal como las ha descripto Perry Anderson (en Consideraciones sobre el marxismo occidental, Madrid, Siglo XXI España, 2012 [1979]), y concebir a las figuras de Altamirano y Sarlo como representantes locales de dicha sub-tradición. sobre el lenguaje; puede ser una actividad carente de utilidad — carente en términos de productividad capitalista_, ligada al ocio, al placer, pero, asimismo, una actividad específica que tiende, al menos en algunos aspectos, a una profesionalización; puede consistir en la expresión de una subjetividad o en la captación de algún fenómeno "externo" y "objetivo"; puede ser concebida como un conjunto reducido de textos encumbrados que sobreviven o como una extensión infinita de ficciones que se despliega hacia el pasado y hacia el futuro; valor de uso y/o valor de cambio; práctica de soledad y/o práctica de socializaciones; mundo de ideas o mundo de materialidades; enunciación contextual y/o enunciación universal; institución y/o mercancía; ámbito autónomo y/o heterónomo; singularidad y/o repetibilidad; ¿Es, para nosotros, a fin de cuentas, algún tipo de fenómeno aprehensible o, más bien, se trata de un ente en huida constante y que siempre escapará a los intentos conceptualizadores y fosilizantes (como esta misma lista de definiciones que aquí consignamos)?. ${ }^{12}$

\section{III}

Entonces, insisto: la sociología de la literatura se nos presenta por medio de una doble problematicidad, derivada de los atributos de lo múltiple, lo complejo y lo opaco que caracterizan tanto a "lo social" como a "lo literario". Ante tal diagnóstico, un programa sensato de la sub-disciplina debería comenzar con esta caución y no con frases altisonantes que anuncian la llegada de la teoría "verdadera", que da por tierra con las precedentes, tal como hace Bourdieu en el pedante y poco meticuloso prólogo de Las reglas del arte (prólogo que nos demuestra, con un éxito pírrico, que un efectismo retórico no implica necesariamente una eficacia argumentativa...)..$^{13}$

Desde luego, como indicaba, a la hora de la investigación práctica, hay que tomar decisiones pragmáticas y operativas. Pero, a veces, tengo la sensación de que algunos investigadores y docentes pasan por alto la doble problematicidad inicial de la sociología de la literatura, sin siquiera tener un mínimo reparo en ella. En este sentido, considero que hay que tomar un principio sociológico de perogrullo y darle vida: no dar por sentadas las cosas, no validar automáticamente los principios y las matrices de pensamiento más afincados, ni los conocimientos más arraigados e internalizados. Aquí me gustaría afirmar una posición durkheimiana irrenunciable, que al día de hoy estructura (jo eso me hicieron creer!) el pensamiento sociológico y de la que la sociología de

12 ¿Y es captable algo del orden de su especificidad? Todorov, en este sentido, es un optimista: "Es preciso mostrar el parecido entre la literatura y los demás sistemas de signos, y al mismo tiempo destacar la originalidad peculiar de la literatura. Pero esto es un trabajo que queda por hacer". Tzvetan Todorov, "Motivo", en Oswald Ducrot y Tzvetan Todorov, Diccionario enciclopédico de las ciencias del lenguaje, Buenos Aires, Siglo XXI, 1974, p. 258

13 Op. cit., pp. 17-75. 
la literatura no puede más que servirse: dejar de lado los preconceptos, los prejuicios y las prenociones. Por supuesto, nunca lo lograremos del todo, pero vale la pena intentarlo y hacer el esfuerzo (si acaso se me acepta la intromisión y la posibilidad de una teoría voluntarista de la acción).

Sí, como buen sociólogo escolar, acabo de citar a Durkheim y a una de sus benditas reglas del método sociológico; pero, llegados a este punto, si tuviera que dar una denominación más precisa a tal exigencia, diría que se trata de considerar el (re) ingreso de la sociología en la sociología de la literatura. Un vector específico de este requerimiento pasa por introducir la teoría sociológica, principalmente en sus formulaciones clásicas, en la sociología de la literatura (tarea que, desde luego, no puede concluir con la insuficiente inclusión de la perspectiva teórica de Bourdieu). ${ }^{14}$ Esto, a su vez, nos lleva a reconocer que una de las posibilidades de dicho reingreso puede derivar en una mera imposibilidad o una desalentadora inutilidad; por lo pronto, habrá que, modestamente, intentarlo.

Por cierto, no niego que los trabajos provenientes de los estudios literarios no se hayan servido en absoluto de la sociología, aunque sí es evidente que sólo han empleado parcelas acotadas de sus desarrollos. Si tuviera que formularlo en una frase sintética, diría: para la sociología de la literatura argentina, heredera de los estudios literarios, Parsons no existe. ${ }^{15}$

\section{IV}

Discuto, a continuación, a partir de dos programas recientes de asignaturas de carreras universitarias (una de posgrado, la otra de grado), que se auto-inscriben — cada una a su manera y en distinta medida - en la tradición de la sociología de la literatura. ${ }^{16}$

En un programa de sociología de la literatura (La Sociología de la

14 No es que esto no se haya hecho en absoluto: pienso, por ejemplo, a propósito del trabajo de Horacio González sobre la picaresca, en su fugaz empleo de los tipos ideales weberianos para formular una reflexión sobre dicho género. Horacio González, La ética picaresca, Montevideo, Altamira, 1992, p. 90.

15 "Parsons", desde luego, como significante que condensa buena parte de la unidad conceptual e institucional de la disciplina sociológica, así como su posterior dispersión, en sintonía con las ideas expuestas con anterioridad por Ricardo Sidicaro, "Las sociologías después de Parsons", en Sociedad, $n^{\circ} 1,1992$, pp. 7-25

16 Discuto a partir de programas de asignaturas, que no es lo mismo que discutir desde la cursada propiamente dicha. Tampoco los programas de una asignatura son equiparables a textos con un mayor grado de elaboración, como artículos o libros. De todas formas, esto no quita que los programas sean textos con un significativo grado de elaboración, en la medida en que en ellos se pondera particularmente la síntesis y la comunicabilidad de distintas perspectivas frente a estudiantes y, justamente por tal condición, se tornan muy pertinentes para el análisis y el debate de la sub-disciplina, pues nos brindan un panorama del programa (de docencia e investigación) de sociología de la literatura que sostiene el docente que lo imparte.
Literatura: Perspectivas Teóricas y Propuestas Metodológicas), elaborado y dictado por Soledad Quereilhac en una maestría de la Universidad Nacional de San Martín en 2018, veo ciertos ejes teóricos ordenados por aportes según autores: Altamirano y Sarlo, Bourdieu, Williams, Jameson, Chartier y Angenot. Se trata de un programa bastante previsible en cuanto a su tradicionalidad -no en sentido peyorativo, sino como una constatación de un linaje de estudios sociales de la literatura dentro de los estudios literarios argentinos-, al punto que, sin contar a Angenot, el mismo podría haber sido presentado, por ejemplo, para un curso de sociología de la literatura en 1998 (¿no pasó nada entre 1998 y 2018?). También me resulta de especial interés el carácter fuertemente conservador en la elección de fuentes literarias para trabajar en clase: Borges, Arlt y Quiroga. Curiosa forma de ver la literatura sin sobrepasar su existencia canónica. ¿No podemos exigirle algo más a un programa de sociología de la literatura? Quisiera creer que sí.

En un programa de sociología del arte (Sociología del Arte: Producción y Circulación de Artes Visuales y Literatura en Argentina), elaborado y dictado por Mariana Cerviño en la carrera de Sociología de la Universidad de Buenos Aires en 2016 (tomo este año, aunque hubo ediciones anteriores y posteriores de la asignatura; la primera vez fue en 2013), tenemos una aproximación a la literatura entendida meramente como prácticas externas, sin que esto suponga ningún tipo de abordaje analítico de las ficciones. Esto, desde luego, responde a una metodología perfectamente válida y con una fuerte tradición teórica: la sociología de orientación bourdieusiana. Sin embargo, el problema que detecto es que, si se enuncia la pretensión de hacer sociología de la literatura e, inmediatamente, se descarta, a lo largo de todo el programa, sin una mínima justificación teórica, la opción de leer ficciones, de hacer lecturas intensivas, entonces no puedo más que evaluar esa actitud —como mínimo- como un descuido bastante inoportuno. Ya sé que leer libros lleva tiempo y que, si uno lee libros de teoría y de literatura (asumiendo, pongamos, que fueran dos cosas de distinto orden), cada una de esas actividades resta tiempo a la otra (benditos economistas y su inexorable costo de oportunidad), pero, ¿esto sería motivo suficiente para descartar de plano el trabajo con ficciones? Quisiera creer que no.

Por lo pronto, entonces, considero que tenemos que ser amplios en el abordaje de "lo social" y "lo literario". Si se me permitiera brindar dos contraejemplos respecto a las observaciones realizadas sobre los programas de las asignaturas de Quereilhac y Cerviño, diría (y no busco ningún tipo de exhaustividad con los siguientes comentarios, que apenas cuentan como pequeñas partículas de discusión): con respecto al primer programa, podríamos efectivamente dar Borges y Arlt, aunque poniendo de manifiesto que nos atenemos a una determinada forma de entender la literatura como un conjunto reducido de textos y de figuras encumbradas, pero que, en paralelo, dicha concepción 
oculta otras prácticas literarias dominadas en el espacio literario, como las traducciones por encargo y las seudotraducciones, por recordar dos de las actividades que analiza Falcón en su riguroso estudio sobre los traductores rioplatenses en la península ibérica - y por pensar en un contraejemplo otorgado por otra persona formada en la misma tradición que Quereilhac-.$^{17}$ De paso: tampoco descartaría empezar cualquier curso de sociología de la literatura con un cuestionamiento sobre cierta asunción tácita de la literatura como unas pocas obras de unas pocas personas a las que prestamos atención. No sólo porque las pocas personas en verdad están en un entramado de muchas personas, sino por el propio carácter distintivo de esos pocos textos a los que prestamos atención, que acarrean, entre otros, el presupuesto de que debemos entender la literatura como el estudio de lo único, lo específico, lo irrepetible y lo innovador, cuando, asimismo, podríamos pensar la literatura, siguiendo una formulación de tintes foucaultianos, como una formación discursiva mediada por un horizonte de "lo decible", así como por iteraciones y no tanto por marcas de unicidad -o incluso por ambas modalidades: las ondas y las ramificaciones de Moretti-.${ }^{18}$ A su vez, creo que Baetens acierta cuando postula la posibilidad de lo literario como un flujo multimodal en que la palabra escrita tiende a perder peso (en otras palabras: ¿vamos a dejar al inconmensurable flujo audiovisual por fuera de "lo literario"? ¿Y en 2018 vamos a dejar de lado los efectos de la ineluctable vida digital?).19

En cuanto a posibles comentarios sobre la asignatura dictada por Cerviño, insisto: un programa que de entrada descarta, sin mucha justificación teórica ni metodológica, la lectura intensiva de textos, incurre en una gran falencia. Esto es independiente de la existencia de tradiciones de pensamiento que enfatizan tal o cual método y se basa en una cuestión previa: hoy en día no se puede desconocer o ignorar otras formulaciones de distintas tradiciones de sociología de la literatura. La indiferencia respecto a otras corrientes de trabajo ajenas a la propia demuestra una gran falta, pero, a fin de cuentas (y con un poco de autocrítica),

17 Alejandrina Falcón, Traductores del exilio. Argentinos en editoriales españolas: traducciones, escrituras por encargo y conflicto lingüístico (1974-1983), Madrid/Fráncfort del Meno, Iberoamericana/Vervuert, 2018.

18 Por cierto, no olvidemos que Moretti, además de matizar que el canon es menos del $1 \%$ de la literatura, dice que es menos del $1 \%$ de la literatura publicada (op. cit., p. 55). En este sentido, por ejemplo, para el caso argentino se hace necesario incorporar el estudio de producciones literarias que no llegan a publicarse. No digo que hacer esto sea fácil, pero si ni siquiera empezamos por visibilizar este fenómeno...

19 Jan Baetens, "World Literature and Popular Literature: Toward a Wordless Literature?", en Theo D'haen, David Damrosch y Djelal Kadir (eds.), The Routledge Companion to World Literature, Abingdon/Nueva York, Routledge, 2012, pp. 336-344. Con respecto a la consideración de internet para un programa de sociología de la literatura, Vanoli nos recuerda que la mayoría de las teorías con que trabajan las ciencias sociales y humanísticas fueron gestadas en un contexto en que nuestras vidas cotidianas no estaban tan permeadas por su presencia. Hernán Vanoli, El amor por la literatura en tiempos de algoritmos. 11 hipótesis para discutir con escritores, editores, lectores, gestores y demás militantes, Buenos Aires, Siglo Veintiuno, 2019, p. 19. se trata de una falta reparable. Creo, en este sentido, que uno de los aspectos más cuestionables del programa de Cerviño (y del de Quereilhac) es cierto sesgo de inclusiones y omisiones de enfoques en vínculo directo con la propia trayectoria, cierto sesgo que deriva en una serie de elecciones autoconfirmantes de la posición del docente a partir de los autores y las corrientes teóricas a tratar (¿por qué no se acepta que puedan dictarse clases sobre temas en los que no seamos especialistas?; este aspecto incluso ayudaría a desmontar la noción del docente como un "iluminado-ilustrado" que debe saber todo y al que nunca se le acepta la siguiente combinación sintagmática: no sé). La asignatura de Cerviño posee una indudable y taxativa orientación bourdieusiana, no sólo a partir de la indiscutible y central presencia de Las reglas del arte, sino también a través de la bibliografía de algunos discípulos de Bourdieu: Boschetti, Casanova, Miceli, Pinto y Sapiro - quienes, por más críticas que efectúen a su mentor, no dejan de ser epígonos-. Se eligen tales autores y corrientes de pensamiento que representan una forma específica de entender la sociología de la literatura según la propia visión del que dicta la materia, pero, ¿por qué no incluir autores y perspectivas con los que estamos en desacuerdo? ¿Por qué no incluir autores y perspectivas cuya existencia no ignoramos, aunque no conozcamos con exactitud sus propuestas teórico-metodológicas? ¿Para qué? Para estudiar, aprender más, discutir, argumentar, contraargumentar y seguir discutiendo. ¿O no se trataba exactamente de eso la labor de las disciplinas sociales y humanísticas? En este punto, siempre me gusta recordar una formulación de la fina prosa de Geertz: la clave de nuestras disciplinas no pasa por perfeccionar un consenso, sino por refinar el debate. ${ }^{20}$ Para esto, ¿qué mejor que incluir perspectivas teóricas y metodológicas que nos resulten ajenas y que, incluso, hasta pueden convencernos de que efectivamente tienen algo significativo para decir sobre nuestros objetos de estudio? En última instancia, en sintonía con la máxima de Geertz, me parece útil parafrasear una sentencia de Garland (a propósito de los debates entre criminólogos): no es que vayamos a discutir para necesariamente ponernos de acuerdo, pero sí, al menos, para estar en desacuerdo con un mayor grado de precisión. ${ }^{21}$

\section{V}

Tomemos un ejemplo internacional (con presencia en la Argentina a través de la editorial Fondo de Cultura Económica): un libro de Sapiro, La sociología de la literatura (usado en la unidad introductoria del programa de docencia de Quereilhac), es una muy buena aproximación a la sub-disciplina, con un gran párra-

20 Clifford Geertz, "Descripción densa: hacia una teoría interpretativa de la cultura", en La interpretación de las culturas, Barcelona, Gedisa, 2003, p. 39.

21 David Garland, Castigo y sociedad moderna. Un estudio de teoría social, México D. F., Siglo XXI, 2005, p. 17. 
fo inicial que condensa dos tradiciones de abordaje. ${ }^{22}$ Luego, la exposición se organiza con un presupuesto teórico que resulta ordenador, aunque no se discute: las teorías sociológicas (bueno, algunas de ellas), seguidas de los momentos de producción, circulación y recepción de la literatura. Por supuesto, se trata de una forma muy útil e intuitiva de pergeñar la estructura del texto. Pero, ¿por qué no ir por problemas? ${ }^{23}$ Con esto no digo que la exposición no sea pertinente (de hecho, uno hasta creería que se trata de una suerte de homenaje y diálogo con la estructura del libro homónimo y antecesor de Escarpit, cuyo índice se organiza de un modo similar — ¿o se trata de una casualidad, si pensamos que Sapiro no quisiera homenajear a un "positivista"?-), aunque creo que todo programa de sociología de la literatura, además de la doble problematicidad en torno a "lo social" y "lo literario", asimismo debe partir de hacer auto-consciente su lugar de observación y enunciación. Por momentos, Sapiro otorga un énfasis exagerado a Bourdieu, pero, ante el hecho no menor de ser una de sus herederas, ¿esto no debería ser declarado explícitamente ante un lector quizá desprevenido? Resulta interesante que, mientras Bourdieu resulta un tanto magnificado en su texto (entre otros logros, se le adjudica la creación del pensamiento relacional, que en todo caso uno tendría más derecho en atribuir a de Saussure, cuando no a alguien incluso anterior), otras figuras apenas cuentan con una mención menor, como ocurre con Lukács (un pensador, por cierto, poco transitado por la tradición sociológica y mayormente reivindicado y leído desde los estudios literarios).

Más allá de las predilecciones teóricas que asoman como fetichismos, el libro de Sapiro se encarga de recopilar distintos aportes y concepciones de la sociología de la literatura, por lo cual uno se pregunta: ¿no habría sido más sensato llamar al libro Sociologías de la literatura ${ }^{24}$ Cuando no, por cierto, Sociologías

22 "La sociología de la literatura tiene por objeto de estudio el hecho literario en tanto hecho social. Esto implica una doble interrogación: sobre la literatura como fenómeno social, del que participan muchas instituciones e individuos que producen, consumen, juzgan las obras; y sobre la inscripción en los textos literarios de las representaciones de una época y de las cuestiones sociales" (Gisèle Sapiro, La sociología de la literatura, Buenos Aires, Fondo de Cultura Económica, 2016, p. 13). Quizá una fórmula más sintética sea aquella elaborada por Escarpit: "el estudio de la literatura en la sociedad y el estudio de la sociedad en la literatura" (op. cit., p. 40). Resulta curioso que Sapiro, en su libro, sólo dedica un par de menciones a Escarpit (en contraste con la iterativa centralidad otorgada a Bourdieu), a quien prejuzga como representante de un enfoque positivista y presociológico (¿quizá sólo leyó Sociología de la literatura de Escarpit y no llegó a los ensayos más maduros de Hacia una sociología del hecho literario - más allá de que este libro esté citado al final del de Sapiro-?).

23 Pienso, por ejemplo, en el excelente libro de Culler, a propósito del abordaje de los estudios literarios a partir de problemas y no de escuelas de pensamiento. Jonathan Culler, Breve introducción a la teoría literaria, Barcelona, Crítica, 2004

24 Si Sidicaro plantea que, después de Parsons, la sociología existe en plural (op. cit., pp. 7-25), ¿no deberíamos sostener lo mismo a propósito de la sociología de la literatura que, además, aglomera distintas tradiciones provenientes de los estudios literarios? Algo así ya concluía Escarpit hace varios años, cuando afirma que "no sería adecuado hablar por el momento de una sociología de la literatura" (op. cit., p. 43), en una sentencia a la que parece haber sido indiferente su compatriota Sapiro.. de las literaturas, aunque claramente el uso de plurales no nos hace necesariamente plurales en nuestros abordajes de los fenómenos sociales y literarios. Esto me permite volver a enfatizar un punto: la propia estructura sintáctica de la expresión "sociología de la literatura" parecería poner a la sociología como método y a la literatura como objeto; esto me parece pertinente y fiel a lo que ocurre en algunos casos, aunque, a modo de contraste, no dejaría de notar que hay modalidades de la sociología de la literatura que serían, por ejemplo: literatura de la literatura, sociología de la sociología o literatura de la sociología (asumiendo al primer término como método y al segundo como objeto, lo que, por supuesto, acarrea un linaje teórico que tampoco debería agotar las opciones epistemológicas y metodológicas de un programa de sociología de la literatura que no renuncie a cierta versatilidad). ${ }^{25}$

Las concepciones de una sociología de la literatura reduccionista que tienden a ignorar ora la sociología, ora el análisis literario (como son muestras respectivas de cada caso, según veo, los dos programas de docencia expuestos), por lo tanto, me parecen dos formas muy débiles de dar comienzo a un proyecto de indagación, pues parecen recaer en cierto grado de hermetismo cognitivo y de dogmatismo auto-confirmante. Si se me permite una modesta opinión, creo que una amplitud bien argumentada, que no excluya arbitrariamente ciertas corrientes teóricas y metodológicas, implicaría un punto de inicio más sólido para las bases de la sub-disciplina.

Entonces, insisto: un programa sensato de sociología de la literatura (me permito seguir en singular) debería incluir una enunciación auto-consciente de su forma de posicionarse, de modo que permita hacer explícito su particular enfoque, sus ventajas, sus deficiencias, pero, especialmente, que se trate de un programa comunicable, conmensurable y que se auto-inscriba en un panorama más amplio (panorama más amplio que no todos reconocen ni se molestan en describir a grandes rasgos y que aquí sí nos interesa dejar consignado, al menos de manera un tanto rudimentaria). ${ }^{26}$

VI

La mención a la necesidad de la auto-conciencia de sí y del lugar

25 Desde luego, en este punto entramos en un terreno clasificatorio cuya nomenclatura depende de cada pensador. Por ejemplo, recordemos que Garasa diferencia la sociología de la literatura de la crítica sociológica (op. cit., p. 18). En cuanto a mi propia clasificación: en otro espacio y momento me explayaré al respecto (y sólo espero que sea una promesa que pueda cumplir).

26 De mi parte, considero que un punto de partida crucial para un proyecto de sociología de la literatura debería ser un texto de Weber: "La «objetividad» cognoscitiva de la ciencia social y de la política social" (en Ensayos sobre metodología sociológica, Buenos Aires, Amorrortu, 1978, pp. 39-101). Espero y deseo, en otro tiempo y espacio, explayarme al respecto (¿estoy haciendo muchas promesas? Yo no prometí nada). 
de enunciación ocupado nos devuelve a aspectos particulares de la sociología y los estudios literarios.

Por el lado de la sociología, la consideración de que mucho de la sociología de la literatura escapa a los aportes más tradicionales de la sociología clásica (en un sentido análogo al que, según Heinich, la sociología del arte se halla escindida con respecto a las formulaciones de la sociología - ya que, según la constatación y el diagnóstico de dicha autora, la labor de los sociólogos del arte se aproxima más a la de los historiadores y críticos del arte-). ${ }^{27}$ Por lo tanto, remarco la necesidad de pensar el reingreso de la sociología en la sociología de la literatura (no digo que sea una tarea sencilla ni con respuestas simples; tampoco digo que sea lo único que haya que hacer en un programa de investigación de sociología de la literatura).

Por el lado de los estudios literarios, creo que otro aspecto problemático sería el gran caudal de trabajos que, si bien no se auto-reconocen dentro de la tradición de la sociología de la literatura, no cabrían dudas de que son inscribibles en ella. Básicamente cualquier desarrollo que reflexione sobre algún fenómeno de los extensos dominios literarios en relación con el impreciso espectro de los fenómenos sociales podría reclamar para sí tal derecho, del mismo modo que, sin que lo pidiera, podríamos someterlo al violento arte de la clasificación e incluirlo dentro de la sub-disciplina.

En el caso específico de la sociología de la literatura en la Argentina, se trata de una sub-disciplina con una tradición que tuvo como catalizador a los estudiosos de la literatura (y no a los sociólogos o, al menos, no en sus orígenes). ${ }^{28}$ De modo que las referencias teóricas de la sociología de la literatura argentina no suelen apelar a los puntales teóricos de la sociología clásica (en un sentido similar al que, como acabamos de señalar, Nathalie Heinich indica que, al menos hasta hace unos años, la sociología del arte casi no se sirve de los aportes centrales de la sociología tradicional). Así, hay nombres de universitarios formados en estudios literarios que son, sin dudas, fundacionales para la tradición nacional de sociología de la literatura: Prieto, Viñas, Garasa, Altamirano, Sarlo, Ludmer, Gramuglio, Rivera, Ford o Lafforgue - y figuras continuadoras de dicha tradición, como Contreras, Garramuño, Laera, Quereilhac y Falcón, por mencionar algunos

27 Nathalie Heinich, Sociología del arte, Buenos Aires, Nueva Visión, 2010, pp. 5-7.

28 Pablo Alabarces destaca el lugar doblemente periférico de la sociología de la cultura (y la literatura) en el panorama de la sociología argentina de mediados y fines del siglo XX: "periferia de la sociología argentina en las ciencias sociales occidentales, pero también de la investigación cultural en la sociología local". Pablo Alabarces, "La cultura y la periferia: andanzas nómades de la sociología de la cultura argentina", en VII Jornadas de Sociología, La Plata, Universidad Nacional de La Plata, 2012, p. 1. Por supuesto, el contexto ha variado en el siglo XXI, en que los investigadores, los proyectos acreditados y la producción académica en el área de la sociología de la cultura cuenta con un significativo reconocimiento, tanto en la sociología como en los estudios literarios (y en el espacio más amplio de las disciplinas sociales y humanísticas). ejemplos-..$^{29}$

Del lado de los sociólogos, cabe traer a cuenta la colaboración conjunta entre Blanco y Jackson, que, si se me permite la etiquetación, diría que es más bien de historia comparada de la sociología antes que de sociología de la literatura —aunque, valgan los rodeos, aquella, en algunas de sus formulaciones, es sin dudas pertinente para esta última_- ${ }^{30}$ Durante la segunda década del siglo XXI, resulta llamativo un conjunto — reducido, pero muy significativo- de desarrollos elaborados por tesistas con formación de base en sociología. A continuación, me refiero a algunos de ellos; no obstante, en paralelo, no debemos dejar de recordar el elevado caudal de tesinas de la carrera de Ciencias de la Comunicación de la Universidad de Buenos Aires que trabajan con fuentes literarias y con temas cercanos a la sociología de la literatura (esta carrera, junto con Sociología, diría que son las dos que cuentan con más aportes al terreno de la sociología de la literatura desde la Facultad de Ciencias Sociales de la Universidad de Buenos Aires, aunque ciertamente existen algunos desarrollos en el ámbito de Ciencia Política que tampoco deberíamos ignorar). ${ }^{31}$ Pienso, entonces, en algunos jóvenes - y no tan jóvenes- sociólogos que escribieron tesis sobre fenómenos literarios y afines: por ejemplo, las tesis de Vanoli y Szpilbarg sobre el mercado editorial y su funcionamiento. ${ }^{32}$ Pienso, asimismo, en dos tesis

29 La lista es híper cuestionable por ser, justamente, una lista (y peor: una lista que depende de mis paupérrimas sinapsis). Entre varios auto-reparos, también les daría lugar a nombres que históricamente se desempeñan en las cátedras de teoría literaria (cuyas formulaciones y preguntas resultan muy próximas a algunas de la sociología de la literatura), de Panesi (Jorge Panesi, Críticas, Buenos Aires, Norma, 2000) a Topuzian (Marcelo Topuzian, Muerte y resurrección del autor [1963-2005], Santa Fe, Ediciones de la Universidad Nacional del Litoral, 2014b), entre otros -y para una reflexión reciente sobre los debates en el marco de la teoría literaria, me remito a un sintético artículo de Fernando Bogado, "Los finales de la teoría: investigación y refundación" (en Filología, n 50, 2020, pp. 5-16); para un repaso sobre la historia de la teoría literaria en la Universidad de Buenos Aires, a una serie de artículos publicados en la revista Luthor, cuya octava entrega (Fernando Bogado, Juan Manuel Lacalle y Mariano Vilar, "Aproximaciones a la historia de la Teoría Literaria en la carrera de Letras de la UBA. Parte VIII [2000-2019]", en Luthor, $n^{\circ} 41,2019$, pp. 20-37) contiene hipervínculos para, a su vez, acceder a las etapas previas (todas de lectura sumamente recomendable)-. $\mathrm{Ni}$ hablar si abrimos la lista a filósofos, historiadores, arquitectos y otros profesionales que, con absoluto derecho, podrían reprocharme el lugar marginal que les reservo al final de una nota al pie.

30 Alejandro Blanco y Luiz Carlos Jackson, Sociología en el espejo. Ensayistas, científicos sociales y críticos literarios en Brasil y en la Argentina (1930-1970), Bernal, Universidad Nacional de Quilmes, 2015. De particular interés resulta el tercer capítulo, "Los escenarios de la crítica", pp. 175-236, en que se reponen y estudian las trayectorias de dos críticos literarios de vital importancia para la Argentina y Brasil: Adolfo Prieto y Antonio Candido, respectivamente.

31 La extensa lista de títulos de tesinas de Comunicación puede consultarse a través del sitio web de la carrera: http://comunicacion.sociales.uba.ar/. Desde luego, muchos de estos trabajos son inscribibles en la tradición de la sociología de la literatura proveniente de los estudios literarios (recordemos que buena parte del primer plantel docente de la Licenciatura en Ciencias de la Comunicación incluía a graduados de la carrera de Letras).

32 Hernán Vanoli, Por una sociología del espacio editorial: cuatro modelos de edición literaria en la Argentina del siglo XX. Tesis de maestría, Buenos Aires, Universidad de Buenos Aires, 2010; Daniela Szpilbarg, Las tramas de la edición mundializada: transformaciones y horizontes del campo editorial en Argentina (1998-2013), Tesis doctoral, Buenos Ai- 
doctorales que exponen dos formas tan distintas como válidas de practicar la sociología de la literatura: por un lado, la de Stegmayer sobre las formas de la violencia en un corpus de narrativa argentina contemporánea; por otro, la de Riveiro sobre la trayectoria y la centralidad de Aira en el campo literario argentino. ${ }^{33}$ Estas dos tesis podrían leerse como representantes locales de esa disputa entre dos programas de sociologías de la literatura que mencionamos al principio (incluso cuando, en el caso de Stegmayer, no haya una explícita auto-inscripción dentro de la sociología de la literatura): se leen textos o se analizan prácticas (sin que, como ya hemos dicho, una vía anule la otra). Combinarlos (o no) es un trabajo que eventualmente cada investigador mide y evalúa en torno a la especificidad de su propio objeto de estudio y a las preguntas que se formula, pero creo que cualquier elección debería tener una fina y explícita justificación tanto de sus asunciones como de sus rechazos teóricos y metodológicos, dado el amplio panorama de abordajes que puede incluir un programa de esta sub-disciplina.

Por último, así como acabo de marcar una diferencia sustantiva entre las tesis doctorales de las sociólogas Stegmayer y Riveiro, tampoco puedo evitar señalar un punto en común: ambas trabajan sobre fracciones legítimas y/o canónicas de la literatura. Ante este diagnóstico, vuelven a cobrar interés enfoques que estudian producciones y consumos no legítimos e incluso vergonzantes (o que al menos así son valorados desde ciertas posiciones dominantes de la cultura literaria), como la investigación de Tennina sobre el Movimiento de Literatura Marginal en Brasil o la de Felitti y Spataro sobre las lectoras de Cincuenta sombras de Grey — y el resto de la saga a la que dicha novela da comienzo-.$^{34}$ ¿Qué tienen de interesante estos trabajos? Muchas cosas, pero déjenme quedarme con una: ponen en jaque el estatuto de "lo

res, Universidad de Buenos Aires, 2015. Esta última ha publicado su tesis en la forma de libro, al que ya hemos hecho alusión: Cartografía argentina de la edición mundializada. Modos de hacer y pensar el libro en el siglo XXI. En cuanto a Vanoli, por cierto, su mención nos conduce a su más reciente ensayo, al que también ya nos hemos referido en una nota precedente: El amor por la literatura en tiempos de algoritmos. $\mathbf{1 1}$ hipótesis para discutir con escritores, editores, lectores, gestores y demás militantes. Otro libro recientemente publicado por una socióloga interesada en el cruce entre orden social y literatura es: Oriana Seccia, ¿Clase media? Ensayos de literatura y sociedad desde Gino Germani a la nueva narrativa argentina, Buenos Aires, Ubu Ediciones, 2019 (libro que, por cierto, también proviene de una tesis doctoral). Una última mención la podemos referir a otro sociólogo y tesista, a raíz de un breve libro ensayístico que no es su tesis y que consiste en reflexiones sobre distintos escritores y obras: Pedro Yagüe, Engendros, Buenos Aires, Hecho Atómico, 2018

33 María Stegmayer, Zonas de inquietud: poder, violencia y memoria en la literatura argentina contemporánea (1995-2010), Tesis doctoral, Buenos Aires, Universidad de Buenos Aires, 2012; María Belén Riveiro, La trayectoria de César Aira: la conformación de un centro descentrado en el campo literario de la ciudad de Buenos Aires (1981-2001), Tesis doctoral, Buenos Aires, Universidad de Buenos Aires, 2020

34 Lucía Tennina, ¡Cuidado con los poetas! Literatura y periferia en la ciudad de São Paulo, Rosario, Beatriz Viterbo, 2017; Karina Felitti y Carolina Spataro, "Circulaciones, debates y apropiaciones de Cincuenta sombras de Grey en la Argentina", en Estudios de Género de El Colegio de México, $n^{\circ} 4,2018$, pp. 1-31.
Literario", en contraste con otras investigaciones en que la literatura se presenta como un objeto estable y auto-evidente (como sucede con muchas indagaciones de docentes e investigadores para quienes la literatura sigue siendo un corpus reducido de autores y obras en soporte escrito, más allá de las dinámicas de incorporaciones, recambios y/o permanencias de nombres en el pedestal de los consagrados). De tal forma, considero que este tipo de abordajes deberían ser centrales para un proyecto de una sociología de la literatura versátil -y, por si cabe la aclaración, rescato estos dos trabajos por su reciente aparición, sin que esto suponga soslayar otros dos antecedentes fundamentales en los estudios críticos sobre literaturas marginales y/o no legítimas, como son los ya clásicos aportes de Sarlo y Prieto, publicados por primera vez en la década de 1980, sobre narraciones sentimentales y criollismo, respectivamente- ${ }^{35}$

Así como, en el final de sus "Conjeturas sobre la literatura mundial", Moretti dice que el motivo de existencia de la literatura comparada es ser una espina para el estudio de las literaturas nacionales, ${ }^{36}$ en cierto movimiento análogo me gustaría cerrar con la siguiente afirmación: la sociología de la literatura debe ser una espina para cualquier investigación y desarrollo de conocimiento que se atenga a actitudes herméticas, tanto en sus concepciones sobre "lo literario" como sobre "lo social". La sociología de la literatura, por lo tanto, debe ser una espina doble: para los estudios literarios y para la sociología.

Quisiera discutir estos puntos sobre sociología de la literatura.

\section{Bibliografía}

Alabarces, Pablo, "La cultura y la periferia: andanzas nómades de la sociología de la cultura argentina", VII Jornadas de Sociología, La Plata, Universidad Nacional de La Plata, 2012.

Altamirano, Carlos y Beatriz Sarlo (eds.), Literatura y sociedad, Buenos Aires, Centro Editor de América Latina, 1977.

Altamirano, Carlos y Beatriz Sarlo, Conceptos de sociología literaria, Buenos Aires, Centro Editor de América Latina, 1980.

Altamirano, Carlos y Beatriz Sarlo, Literatura/Sociedad, Buenos Aires, Hachette, 1983.

Anderson, Perry, Consideraciones sobre el marxismo occidental, Madrid, Siglo XXI España, 2012 [1979].

35 Beatriz Sarlo, El imperio de los sentimientos. Narraciones de circulación periódica en la Argentina, 1917-1925, Buenos Aires, Siglo XXI, 2011; Adolfo Prieto, El discurso criollista en la formación de la Argentina moderna, Buenos Aires, Sudamericana, 1988.

36 Op. cit., p. 68. 
Baetens, Jan, "World Literature and Popular Literature: Toward a Wordless Literature?", en Theo D'haen, David Damrosch y Djelal Kadir (eds.), The Routledge Companion to World Literature, Abingdon/Nueva York, Routledge, 2012, pp. 336-344.

Baranger, Denis, "La recepción de Bourdieu en Argentina", en Desarrollo Económico, n 197, 2010, pp. 129-146.

Barthes, Roland, y otros, Literatura y sociedad. Problemas de metodología en sociología de la literatura, Barcelona, Martínez Roca, 1969.

Blanco, Alejandro, y Luiz Carlos Jackson, Sociología en el espejo. Ensayistas, científicos sociales y críticos literarios en Brasil y en la Argentina (1930-1970), Bernal, Universidad Nacional de Quilmes, 2015.

Bogado, Fernando, "Los finales de la teoría: investigación y refundación", en Filología, n50, 2020, pp. 5-16.

Bogado, Fernando, Juan Manuel Lacalle y Mariano Vilar, "Aproximaciones a la historia de la Teoría Literaria en la carrera de Letras de la UBA. Parte VIII (2000-2019)", en Luthor, n 41, 2019, pp. 20-37.

Bourdieu, Pierre, Las reglas del arte. Génesis y estructura del campo literario, Barcelona, Anagrama, 2005.

Casanova, Pascale, La República mundial de las Letras, Barcelona, Anagrama, 2001.

Cerviño, Mariana, "Sociología del Arte: Producción y Circulación de Artes Visuales y Literatura en Argentina". Programa de asignatura optativa de la Licenciatura en Sociología, Facultad de Ciencias Sociales, Universidad de Buenos Aires, 2016.

Cevasco, María Elisa, "Literatura, sociología de la", en Carlos Altamirano (dir.), Términos críticos de sociología de la cultura, Buenos Aires, Paidós, 2002, pp. 161-167.

Culler, Jonathan, Breve introducción a la teoría literaria, Barcelona, Crítica, 2004

Dujovne, Alejandro, y Diego García, "Introducción a la 'Literatura mundial'", en Políticas de la Memoria, n 10/11/12, 2009-2011, pp. 31-37.

Escarpit, Robert, Sociología de la literatura, Buenos Aires, Fabril Editora, 1962.

"Lo literario y lo social", en Robert Escarpit y otros, Hacia una sociología del hecho literario, Madrid, Edicusa, 1974, pp. 1143.
"Éxito y supervivencia literarios", en Robert Escarpit y otros, Hacia una sociología del hecho literario, Madrid, Edicusa, 1974, pp. 131-164.

Falcón, Alejandrina, Traductores del exilio. Argentinos en editoriales españolas: traducciones, escrituras por encargo y conflicto lingüístico (1974-1983), Madrid/Fráncfort del Meno, Iberoamericana/Vervuert, 2018.

Felitti, Karina, y Carolina Spataro, "Circulaciones, debates y apropiaciones de Cincuenta sombras de Grey en la Argentina", en Estudios de Género de El Colegio de México, n 4, 2018, pp. 1-31.

Garasa, Delfín, Literatura y sociología, Buenos Aires, Troquel, 1973.

Garland, David, Castigo y sociedad moderna. Un estudio de teoría social, México D. F., Siglo XXI, 2005.

Geertz, Clifford, "Descripción densa: hacia una teoría interpretativa de la cultura", en La interpretación de las culturas, Barcelona, Gedisa, 2003.

Goldmann, Lucien, y otros, Sociología de la creación literaria, Buenos Aires, Nueva Visión, 1971.

Goldmann, Lucien, Para una sociología de la novela, Madrid, Ayuso, 1975.

González, Horacio, La ética picaresca, Montevideo, Altamira, 1992.

Gramuglio, María Teresa, "El cosmopolitismo de las literaturas periféricas", en CeLeHis. Revista del Centro de Letras Hispanoamericanas, $n^{\circ} 19,2008$, pp. 159-172

"Literatura argentina y literaturas europeas. Aproximaciones a una relación problemática", CeLeHis. Revista del Centro de Letras Hispanoamericanas, $n^{\circ} 16,2004$, pp. 11-27

Heinich, Nathalie, Sociología del arte, Buenos Aires, Nueva Visión, 2010.

Luhmann, Niklas, Sistemas Sociales: lineamientos para una teoría general, Rubí (Barcelona)/México D. F./Bogotá, Anthropos/Universidad Iberoamericana/Pontificia Universidad Javeriana, 1998.

Lukács, György, Sociología de la literatura, Madrid, Península, 1966.

Martínez, Ana Teresa, "Lecturas y lectores de Bourdieu en la Ar- 
gentina", en Prismas: revista de historia intelectual, $n^{\circ} 11,2007$, pp. 11-30.

Moretti, Franco, "Conjectures on World Literature", en New Left Review, $n^{\circ} 1,2000$, pp. 54-68.

Panesi, Jorge, Críticas, Buenos Aires, Norma, 2000.

Prieto, Adolfo, El discurso criollista en la formación de la Argentina moderna, Buenos Aires, Sudamericana, 1988.

Quereilhac, Soledad, "La Sociología de la Literatura: Perspectivas Teóricas y Propuestas Metodológicas". Programa de asignatura optativa de la Maestría en Sociología de la Cultura y Análisis Cultural, Instituto de Altos Estudios Sociales, Universidad Nacional de San Martín, 2018.

Riveiro, María Belén, La trayectoria de César Aira: la conformación de un centro descentrado en el campo literario de la ciudad de Buenos Aires (1981-2001), Tesis doctoral, Buenos Aires, Universidad de Buenos Aires, 2020.

Santoro, Marco, "Putting Bourdieu in the Global Field. Introduction to the Symposium", en Sociologica, $n^{\circ} 2,2008$, pp. 1-32.

Sapiro, Gisèle, La sociología de la literatura, Buenos Aires, Fondo de Cultura Económica, 2016.

Sarlo, Beatriz, El imperio de los sentimientos. Narraciones de circulación periódica en la Argentina, 1917-1925, Buenos Aires, Siglo XXI, 2011

Seccia, Oriana, ¿Clase media? Ensayos de literatura y sociedad desde Gino Germani a la nueva narrativa argentina, Buenos $\mathrm{Ai}$ res, Ubu Ediciones, 2019

Sidicaro, Ricardo, "Las sociologías después de Parsons", en Sociedad, $n^{\circ} 1,1992$, pp. 7-25.

Stegmayer, María, Zonas de inquietud: poder, violencia y memoria en la literatura argentina contemporánea (1995-2010), Tesis doctoral, Buenos Aires, Universidad de Buenos Aires, 2012.

Szpilbarg, Daniela, Las tramas de la edición mundializada: transformaciones y horizontes del campo editorial en Argentina (1998-2013), Tesis doctoral, Buenos Aires, Universidad de Buenos Aires, 2015.

Cartografía argentina de la edición mundializada. Modos de hacer y pensar el libro en el siglo XXI, Temperley, Tren en Movimiento, 2019.
Tabarovsky, Damián, Literatura de izquierda, Rosario, Beatriz Viterbo, 2004

Tennina, Lucía, ¡Cuidado con los poetas! Literatura y periferia en la ciudad de São Paulo, Rosario, Beatriz Viterbo, 2017.

Todorov, Tzvetan, "Motivo", en Oswald Ducrot y Tzvetan Todorov, Diccionario enciclopédico de las ciencias del lenguaje, Buenos Aires, Siglo XXI, 1974, pp. 254-258.

Topuzian, Marcelo, "La literatura mundial como provocación de los estudios literarios", en Chuy. Revista de estudios literarios latinoamericanos, $n^{\circ} 1,2014 a$, pp. 94-138.

Muerte y resurrección del autor (1963-2005), Santa Fe Ediciones de la Universidad Nacional del Litoral, 2014b.

"Introducción: entre literatura nacional y posnacional", en Marcelo Topuzian (comp.), Tras la nación: conjeturas y controversias sobre las literaturas nacionales y mundiales, Buenos Aires, Editorial Universitaria de Buenos Aires, 2017, pp. 9-65.

Vanoli, Hernán, "Pequeñas editoriales y transformaciones en la cultura literaria argentina", en Apuntes de Investigación del CECYP, n 15, 2009, pp. 161-185.

Por una sociología del espacio editorial: cuatro modelos de edición literaria en la Argentina del siglo XX, Tesis de maestría, Buenos Aires, Universidad de Buenos Aires, 2010.

El amor por la literatura en tiempos de algoritmos. 11 hipótesis para discutir con escritores, editores, lectores, gestores y demás militantes, Buenos Aires, Siglo XXI, 2019.

Weber, Max, "La «objetividad» cognoscitiva de la ciencia social y de la política social", en Ensayos sobre metodología sociológica, Buenos Aires, Amorrortu, 1978, pp. 39-101.

Yagüe, Pedro, Engendros, Buenos Aires, Hecho Atómico, 2018. 


\section{Resumen}

En el marco de una sociología de la literatura argentina que ha sido desarrollada principalmente por representantes de los estudios literarios, planteamos una discusión sobre los puntos de partida de la sub-disciplina. Para esto, elaboramos una argumentación centrada en la necesidad de sentar unas bases relativamente amplias para un programa de investigación y docencia en sociología de la literatura. Remarcamos dos requisitos: por un lado, la sensatez de partir de una gran doble duda basada en la intransparencia de "lo social" y "lo literario"; por otro, la necesidad de hacer explícito el propio lugar de enunciación y observación. Discutimos con enfoques teóricos de distintos autores (Bourdieu, Casanova, Escarpit, Moretti y otros), así como con dos programas de docencia universitaria de sociología de la literatura. Además, pasamos revista a un somero panorama nacional de aportes desde los estudios literarios y la sociología. Buscamos, por sobre todas las cosas, discutir, por lo que no arribamos a ningún tipo de conclusión "superadora", aunque sí afirmamos que un programa de sociología de la literatura deberá ser una constante molestia, tanto para los estudios literarios como para la sociología.

Palabras clave: Sociología de la literatura; Sociología; Teoría sociológica; Estudios literarios; Literatura

\section{Abstract}

\section{Discussion on Sociology of Literature}

Within the framework of an Argentine sociology of literature that has been developed mainly by representatives of literary studies, I propose a discussion on the starting points of the sub-discipline. For this, I develop an argument focused on the need to lay relatively broad foundations for a research and teaching program in sociology of literature. I highlight two requirements: on the one hand, a starting point from a great double doubt based on the intransparence of "the social" and "literature"; on the other, the need to make explicit the place of enunciation and observation itself. I discuss with theoretical approaches of different authors (Bourdieu, Casanova, Escarpit, Moretti and others), as well as with two university teaching programs of sociology of literature. In addition, I review a national panorama of contributions from literary studies and sociology. I promote, above all things, discussion, so I do not arrive at any kind of "overcoming" conclusion, although I do affirm that a program of sociology of literature must be a constant nuisance, both for literary studies and for sociology.

Keywords: Sociology of Literature; Sociology; Sociological Theory; Literary Studies; Literature

Recibido: 20-12-2019

Aceptado: 21-7-2020 